\title{
PERIASTRON OBSERVATIONS OF THE
}

\author{
PSR B1259-63/SS 2883 BINARY SYSTEM
}

\author{
SIMON JOHNSTON \\ Research Centre for Theoretical Astrophysics \\ University of Sydney, NSW 2006, Australia.
}

\section{Introduction}

PSR B1259-63 is a 47-millisecond pulsar which was discovered in a high frequency survey of the galactic plane (Johnston et al. 1992a) and was subsequently found to be in a highly eccentric orbit with a main-sequence Be star known as SS 2883 (Johnston et al. 1992b). Radio observations of the pulsar led to a phase connected timing solution which predicted the epoch of periastron to be 1994 January 9 (MJD 49361.2); optical observations of the Be star led to a determination of its mass and of the size of its circumstellar disk (Johnston et al. 1994a): the star is of approximate spectral type B1e, with mass $10 \mathrm{M}_{\odot}$ and radius $6 \mathrm{R}_{\odot}$. If this mass is correct and the pulsar has a mass of $1.4 \mathrm{M}_{\odot}$, then the inclination angle of the plane of the orbit with respect to the sky is $35^{\circ}$. This pulsar has an unusually flat radio spectrum compared to most pulsars, which makes it easily detectable up to 8.4 GHz. The narrow pulse permits dispersion and scattering measurements for studying the ionized plasma in the system. Moreover, the pulses are highly linearly polarized and permit determination of the rotation measure (RM), allowing measurements of the magnetic field along the line of sight. The 3.5-yr orbit of the pulsar around its companion thus provides us with an excellent probe of the stellar wind of the Be star over a wide frequency range.

Be stars are generally defined as non-supergiant B-type stars which show one or more of the Balmer lines in emission. These emission lines generally show a double-peaked structure, and it was realized (Struve 1931) that this could be interpreted as originating from a rotating disk of material in the equatorial region of the star. These disks have been observed at optical, infrared, millimeter and radio wavelengths and are generally assumed to 
consist of high-density, slowly moving material which is more or less confined to the equatorial plane of the star. Be stars are also bright UV sources, and observations of UV resonance lines seem to indicate the presence of a more global wind, with low density and high velocity.

\section{Observations and Data Analysis}

All observations of the pulsar were made using the Parkes 64-m radio telescope of the Australia Telescope National Facility in New South Wales, Australia. Four separate dual-channel cryogenic receiver systems were used at frequencies of $1.5,2.3,4.8$ and $8.4 \mathrm{GHz}$. Each receiver was sensitive to two orthogonal planes of linear polarization and had a probe injecting a linearly polarized calibration signal at 45 degrees to the signal probes. Flux density calibration was carried out on Hydra A (PKS 0915-118) for which we assumed flux densities of $42,24,14$ and $8 \mathrm{Jy}$ at each of the four observing frequencies, giving system equivalent flux densities of $40,80,80$ and $130 \mathrm{Jy}$ on cold sky, respectively. The back end consisted of a filter bank for each polarization of 64 individual contiguous sub-bands each $5 \mathrm{MHz}$ wide. The outputs of each sub-band were separately detected to produce the intensities of the two orthogonal polarizations and also multiplied with phase shifts of $\pm 45^{\circ}$ to provide all four Stokes' parameters. The outputs from each frequency channel were high- and low-pass filtered, sampled every $0.6 \mathrm{~ms}$, one-bit digitised and recorded on magnetic tape for off-line analysis. Typical integration times were 30 minutes, after which the entire feed and receiver package were rotated through 90 degrees; this permits the removal of certain instrumental effects from the polarization data. Calibration of the polarization was carried out with a pulsed calibration noise source and, as a further check, by observations of pulsars with known polarization characteristics.

\section{Results}

In broad terms, our results showed that the pulses became depolarized, at least at low frequencies, as early as 1993 October. The pulsar was not detectable in timing data taken on 1993 November 27 at $1.5 \mathrm{GHz}$ but had re-appeared, although scattered, at this frequency on 1993 December 11. The pulsar then became increasingly scatter-broadened at even the highest frequency, and the last detection of the pulses prior to periastron was made (at $4.8 \mathrm{GHz}$ ) on 1993 December 20. During this period there was evidence for an increase in the dispersion measure (DM) of the pulsar. In spite of extensive observations throughout 1994 January, the pulses remained undetectable. The first detection of the pulses following periastron occurred on 1994 February 2, when the pulses were unscattered even at $1.5 \mathrm{GHz}$. 
The pulse period was close to that predicted from the binary parameters, although phase could not be unambiguously connected across periastron passage. Around this time, the pulses were depolarized at $1.5 \mathrm{GHz}$ and partially polarized at the higher frequencies. By mid April 1994, the DM, the polarization and the RM had returned to the values observed in 1993 August.

The pulsar has a DM of $146.7 \mathrm{~cm}^{-3} \mathrm{pc}$ for most of its orbital period. The first evidence of a change in $\mathrm{DM}$ is from data taken at $1.5 \mathrm{GHz}$ on 1993 November 18. These data show a larger than expected residual from the phase connected solution implying an increase in the value of $D M$ of about $0.4 \pm 0.2 \mathrm{~cm}^{-3} \mathrm{pc}$. On 1993 December 9 from observations at $2.3 \mathrm{GHz}$ and $5 \mathrm{GHz}$, the DM was $150.4 \pm 0.2 \mathrm{~cm}^{-3} \mathrm{pc}$, an increase of $3.7 \mathrm{~cm}^{-3} \mathrm{pc}$ over the nominal DM. The last observations of the pulsed emission from PSR B1259-63 before periastron, taken at $5 \mathrm{GHz}$ on 1993 December 20 showed the DM to have increased to $158.0 \pm 0.2 \mathrm{~cm}^{-3} \mathrm{pc}$. Following periastron, multi-frequency observations on 1994 February 4, showed the DM to be $146.9 \pm 0.2 \mathrm{~cm}^{-3} \mathrm{pc}$, an enhancement of at most $0.4 \mathrm{~cm}^{-3} \mathrm{pc}$. By 1994 March 4, the DM had returned to its nominal value of $146.7 \mathrm{~cm}^{-3} \mathrm{pc}$.

The pulsar was observed in 1993 August as part of a study of the polarization properties of pulsars (Manchester \& Johnston 1994). The pulsar shows about 80 per cent linear polarization at all frequencies from 1.5 to $8.4 \mathrm{GHz}$. The RM at that epoch was $+19 \mathrm{rad} \mathrm{m}^{-2}$. The pulsar was next observed in polarization mode on 1993 October 8 at $1.5 \mathrm{GHz}$, when the linear polarization amounted to less than 2 per cent of the total flux. At this epoch no observations were made at higher frequencies. It was further observed at 2.3, 4.8 and 8.4 GHz from 1993 December 9-11 and no linear polarization was evident at these frequencies. This continued throughout December in spite of an extensive search of the RM parameter space.

Following periastron, and the re-emergence of the pulses, depolarization was still in evidence at $1.5 \mathrm{GHz}$ on 1994 February 2. Four sets of observations were made at $8 \mathrm{GHz}$ on 1994 February 3. The first two have low signal-to-noise ratios and no polarization can be measured. The final two observations revealed a rather stronger signal, and there is evidence for a very large and negative $R M$. The two observations show similar polarization position angles of $70^{\circ}$ and $15^{\circ}$ for the main pulse and the interpulse. This can be compared with the position angles for the data taken on 1994 March $4\left(35^{\circ}\right.$ and $\left.-20^{\circ}\right)$. In spite of the $180^{\circ}$ position angle ambiguities, by looking at the percentage of linear polarization at different RMs we conclude that the best value from the position angle changes and the linear polarization is $-6780 \mathrm{rad} \mathrm{m}^{-2}$. What makes these observations more remarkable is that there is apparently an increase of only $0.2 \mathrm{~cm}^{-3} \mathrm{pc}$ in the $\mathrm{DM}$ of the $1.5 \mathrm{GHz}$ data taken at the same epoch. From the ratio of the RM to the DM we can 
get an estimate of the magnetic field parallel to the line of sight. Using an $\mathrm{RM}$ of $-6800 \mathrm{rad} \mathrm{m}^{-2}$ and a DM of $0.2 \mathrm{~cm}^{-3} \mathrm{pc}$, the magnetic-field strength is $0.2 \mathrm{G}$ at a distance of $\sim 45 \mathrm{R}_{*}$.

The $5 \mathrm{GHz}$ data taken only 5 days (February 8 ) later show remarkable changes in both the the RM and the percentage of linear polarization on short time scales. Twelve 30 minute integrations were made in total. The first half of the data shows no polarization down to a limit of 5 per cent of the total intensity. During the second half of the data, the fractional linear polarization rose steadily and in the final integration was as high as 60 per cent. In the first of the integrations that shows some linear polarization, the $\mathrm{RM}$ was $-1630 \mathrm{rad} \mathrm{m}^{-2}$. This then increased by about $200 \mathrm{rad} \mathrm{m}^{-2}$ before settling at a value around $-1400 \mathrm{radm}^{-2}$ for the rest of the integration. Observations with such a large change in $R M$ in such a short time scale are unprecedented. There were no DM changes measurable in these observations.

On 1994 March 4 polarization observations were again made at three frequencies. At 5 and $8 \mathrm{GHz}$, the pulsar appeared to have the same fractional linear polarization as in 1993 August. In the $1.5 \mathrm{GHz}$ data, however, the linear component was only about 50 per cent. The RM had changed to $-69 \mathrm{rad} \mathrm{m}^{-2}$ as measured across the bandwidth at $1.5 \mathrm{GHz}$, and this change was confirmed by the change in the absolute position angle at 1.5, 5 and 8.4 GHz when compared with the 1993 August data. During these observations there were no changes in the RM over short time scales. By 1994 April 12, linear polarization had been fully restored at $1.5 \mathrm{GHz}$ (measurements were made at this frequency only). The $\mathrm{RM}$ was $25 \mathrm{rad} \mathrm{m}^{-2}$. Further observations on 1994 May 10 gave an RM of $22 \mathrm{rad} \mathrm{m}^{-2}$.

\section{Discussion}

We will attempt to model the equatorial wind or disk of the Be star using a simple model. Waters (1986) derived a numerical model based on IRAS observations of Be stars. He assumed that the electron number density, $n_{\mathrm{e}}$, as a function of radius varied as

$$
n_{e}(r)=n_{0}\left(r / R_{*}\right)^{-\beta}
$$

where $r$ the distance from the star and $n_{0}$ is the density at the radius, $R_{*}$, of the stellar surface. Waters found that $\beta$ typically had values between 2 and 4. Optical observations of the Balmer emission lines from a sample of Be stars (Slettebak et al. 1992) showed that these disks extended to at least a few tens of stellar radii and that $n_{0}$ had values between $10^{11}$ and $10^{13} \mathrm{~cm}^{-3}$. He further assumed that the disk had an opening angle of about $15^{\circ}$ and that the disk was truncated at some radius. Recent radio 
observations of Be stars (Dougherty et al. 1991; Dougherty \& Taylor 1992) showed that the emission can extend as far as a few hundreds of stellar radii and in the case of $\psi$ Persei extends to thousands of stellar radii. While it is thus clearly unphysical to assume an abrupt cut-off to the disk, we assume here that outside the disk the gaseous material contributes only a insignificant amount to the integrated electron density along the line of sight. Throughout the modelling process we assume an orbital inclination of $35^{\circ}$ and that the equatorial plane of the Be star lies in the orbital plane.

We will assume that the magnetic field, $B$, in the wind has the form

$$
B(r)=B_{0}\left(r / r_{*}\right)^{-1}
$$

where $B_{0}$ is the magnetic field strength at the stellar surface. The field thus has a $1 / r$ dependence; this is probably true beyond $5-10 R_{*}$ although inside this radius the field may be dipolar. However, at all orbital phases at which there are meaningful data the line of sight does not come within $10 R_{*}$ so the exact nature of the magnetic field structure within this radius is not important. We will further assume that the galactic contribution to the $\mathrm{RM}$ is $\sim 25 \mathrm{rad} \mathrm{m}^{-2}$ which is consistent with the values measured in 1993 August and after 1994 April.

We can use the polarization data to place limits on the extent of the circumstellar disk. Depolarization occurred in 1993 October and the RM did not stabilize until 1994 April. At these dates, the separation of the pulsar from its companion was about $150 \dot{\mathrm{R}}_{*}$. We thus take this to be the extent of the stellar disk. This is much larger than the extent derived from the optical emission lines (Johnston et al. 1994a), however the cooler, less dense outer portions of the disk are better probed at longer wavelengths such as infra-red. The DM data only show measurable changes much closer to the star, at $100 R_{*}$ before periastron and $60 R_{*}$ after periastron. Such asymmetry is expected because the line of sight traverses a greater region of the disk prior to periastron. Due to the low inclination of the binary orbit, however, this only applies if the opening angle of the disk is large. Even so, the rather small observed DM changes imply a large value of $\beta$ in Eq. (2). The best fit to the DM changes has $n_{0}=4.510^{12} \mathrm{~cm}^{-3}$ and $\beta=4.2$. This electron density compares well to the numbers derived on a large sample of Be stars by Waters et al. (1987) but the value of $\beta$ is higher than most of the stars in their sample. If we assume a slow radial outflow to the disk of $\sim 5 \mathrm{~km} \mathrm{~s}^{-1}$ then this leads to a derived mass loss rate in the disk of $510^{-8} \mathrm{M}_{\odot} \mathrm{yr}^{-1}$. This is a typical value for early-type B stars. However even with an $r^{-4}$ dependence on the electron density, it is hard to produce little or no DM change on 1994 February 2. One possible explanation for this apparent strong asymmetry in the DM changes is that the equatorial plane of the star is not in the same plane as the pulsar orbit. 
Although it is hard to constrain the magnetic field given the very few values of RMs that we obtained it is possible to reproduce the general evolution of the RM with time. For the simple field structure described above we might expect the RM contribution from the system to change sign when the pulsar crosses the line of sight to its companion and indeed the data support this. Also, the very steep increase in the RM in early February is satisfactorily modelled despite the small DM changes at this time. Using the values of $n_{0}$ and $\beta$ obtained above, we derive a magnetic field strength at the stellar surface of $0.8 \mathrm{G}$. If this model is correct, then it implies a very large value of RM, in excess of $25000 \mathrm{rad} \mathrm{m}^{-2}$, in 1993 December. As we have seen, strong depolarization occurs at RMs of 1500 and $7000 \mathrm{rad} \mathrm{m}^{-2}$ at 5 and $8 \mathrm{GHz}$ respectively, so the lack of polarization in the pulses in the 1993 December data is not greatly surprising.

Given this model, and assuming a smooth wind (i.e., no clumping) we are in a position to calculate the optical depth as a function of orbital phase. The optical depth, $\tau$, for free-free radiation is give approximately by

$$
\tau=8.210^{-2} T^{-1.35} \nu^{-2.1} \int_{0}^{L} n_{\mathrm{e}}^{2} \mathrm{~d} l
$$

where $T$ is the temperature in Kelvin, $\nu$ the observing frequency in GHz. If we assume that the temperature in the wind has a constant value of $\sim 10^{4} \mathrm{~K}$ (Waters 1986) then we find that the optical depth is about unity at $1.5 \mathrm{GHz}$ on 1993 December 17 and unity at $5 \mathrm{GHz}$ on 1993 December 23, close to the dates of the last detections at these frequencies. Given our simple model, these values agree well with the observations. On 1993 December 10, the flux density at $1.5 \mathrm{GHz}$ was lower than average and following this date the pulsar was undetectable at $1.5 \mathrm{GHz}$. The flux density at $5 \mathrm{GHz}$ on 1993 December 21 was very low and this was the last detection of the pulsar prior to periastron. Thus it would appear as if both free-free absorption and pulse scatter broadening were responsible for the lack of detection of the pulses through periastron. The model also fits the post-periastron data though not as well as the pre-periastron data. The optical depth decreases below unity on 1994 January 16 at $5 \mathrm{GHz}$ and on 1994 January 22 at $1.5 \mathrm{GHz}$. The observations show that the pulsar was undetectable, as predicted, at $1.5 \mathrm{GHz}$ on January 21 but was not detected at $5 \mathrm{GHz}$ the following day in spite of the fact that the predicted optical depth is only $\sim 0.1$. The pulsar was then detectable at both frequencies on 1994 February 3. The discrepancy between the model and the observations can be explained by a moderate amount of clumping in the wind. This may also explain the non-detection of pulsed emission on 1993 November 18 at which time the optical depth from the model was low. 


\section{Conclusions}

Between 1993 August and 1994 May, a time span which encompasses the periastron passage on 1994 January 9 , we have carried out extensive multifrequency radio observations of PSR B1259-63. Using a basic model for the structure and form of a Be star's disk (Waters 1986), which assumes a finite disk with an opening angle $\theta$ and a power law radial dependence on the electron density and magnetic field, we have been able to model the largescale features of the observations. A full report on these observations and their implications will be given in the papers by Johnston et al. (1994b), Manchester et al. (1994) and Melatos et al. (1994).

\section{References}

Dougherty, S.M. \& Taylor, A.R. 1992, Nat 359, 808

Dougherty, S.M., Taylor, A.R. \& Waters, L.B.F.M. 1991, A\&A 248, 175

Johnston, S. et al. 1992a, MNRAS 255, 401

Johnston, S. et al. 1992b, ApJ 387, L37

Johnston, S. et al. 1994a, MNRAS 268, 430

Johnston, S. et al. 1994b, MNRAS (submitted)

Manchester, R.N. \& Johnston, S. 1994, ApJ (submitted)

Manchester, R.N. et al. 1994, ApJ (submitted)

Melatos, A., Johnston, S. \& Melrose, D.B. 1994, MNRAS (submitted)

Slettebak, A., Collins, G.W. \& Truax, R. 1992, ApJS 81, 335

Struve, O. 1931, ApJ 73, 94

Waters, L.B.F.M. 1986, A\&A 162, 121

Waters, L.B.F.M., Coté, J. \& Lamers, H.J.G.L.M. 1987, A\&A 185, 206 